Validity of the diagnosis of personality disorder in adults with learning disability and severe

\title{
behavioural problems
}

\author{
Preliminary study \\ ANDREW FLYNN, HELEN MATTHEWS and SHEILA HOLLINS
}

\begin{abstract}
Background Personality disorder in people with learning disability has received little research attention, with only a handful of cross-sectional surveys of prevalence available. As yet, there have been no studies to include an examination of validity.
\end{abstract}

\begin{abstract}
Aims To investigate the prevalence of personality disorder in adults with learning disability who are in specialist challenging behaviour in-patient services and to examine the validity of the diagnosis of personality disorder in this group in terms of its association with abusive experience in early life.
\end{abstract}

Method The Standardised Assessment of Personality (SAP) was used to diagnose personality disorder in 36 individuals with mild/moderate learning disability. Case notes were reviewed for details of clinical diagnosis and early psychosocial history.

Results Thirty-nine per cent of the sample met the criteria for severe personality disorder. This diagnosis showed a significant association with early traumatic experience.

\section{Conclusions Severe personality} disorder is a common diagnosis in this group. There is preliminary evidence that the diagnosis is associated with abuse in childhood.

Declaration of interest Support from Partnerships in Care.
In contrast to other psychiatric diagnoses in adults with learning disabilities, personality disorder has received little attention. A small number of cross-sectional surveys of prevalence have established the reliability of the Standardised Assessment of Personality (SAP; Pilgrim et al, 1990) for adults with learning disability and found high rates of the diagnosis in hospital and community settings (Ballinger \& Reid, 1987; Khan et al, 1997). However, although the diagnostic criteria can be applied reliably, their validity in terms of aetiology, prognosis and treatment response has not been researched. This study examines the prevalence of severe personality disorder in an in-patient population with severe challenging behaviour and attempts to demonstrate preliminary evidence for the validity of the diagnosis in terms of aetiology. The results have important implications for further research and the development of clinical services.

\section{METHOD}

\section{Participants}

Patients from four in-patient psychiatric services for adults with learning disabilities and severe challenging behaviour were invited to participate in the study. Approval was obtained from the local research ethics committee for each centre.

The responsible psychiatrist for each service identified all individuals with mild or moderate learning disability who had no clinical contraindication for research participation. Individuals were recruited if they gave informed consent and could identify an informant who had known them well over the previous 5 years.

\section{Instruments and data collection}

The Standardised Assessment of Personality (SAP; Pilgrim et al, 1990) is a semistructured diagnostic interview. Ballinger \& Reid (1987) have shown it to have satisfactory interrater reliability when used with people with learning disabilities in an institutional setting. It is administered by a trained interviewer to an informant who has at least 5 years of acquaintance with the participant. The interview generates ICD-10 (World Health Organization, 1992) diagnoses of personality disorder. The guidance recommends that where more than one category can be assigned, the 'most disabling' should be rated alone. However, because of the preliminary nature of this study and the desire to avoid unnecessary subjectivity, in cases where multiple diagnoses were made, each was accorded equal rank.

Case notes were reviewed by the principal investigator (A.F.) for clinical diagnoses and histories of childhood abuse or neglect. Although this judgement was largely subjective, instances had to be associated with a child protection response by social services. These reviews were carried out blind to the results of SAP ratings.

\section{RESULTS}

\section{The SAP assessments}

Thirty-six SAP assessments and case-note reviews were completed across four inpatient units. These units ranged in size from 12 to 40 beds and had patients with all degrees of learning disability (though mostly mild to moderate) with a variety of psychiatric diagnoses. All of those resident on these units had severe behavioural problems, especially self-harm and violence to others, whose needs could not be met by community services. The 36 interviews represented $46 \%$ of all residents on these units; 27 interviews were with hospital staff and the remainder were with parents. Of those taking part, 25 were male and 11 female.

The SAP diagnoses of personality disorder could be assigned in 34/36 (92\%) of cases. Co-occurrence of personality disorder categories was common, with a mean of 3.4 per person (range $0-8$ ). The frequencies of personality disorder diagnoses, as derived from the SAP, are shown in Table 1.

\section{Personality disorder and early traumatic experience}

It was hypothesised that if personality disorder is a valid diagnosis in learning disability, then it should show some evidence 
Table I Frequencies of Standardised Assessment of Personality (SAP) diagnoses of personality disorder in the 36 participants

\begin{tabular}{lcc}
\hline SAP category & $\begin{array}{c}\text { No. of times } \\
\text { diagnosed }\end{array}$ & $\begin{array}{c}\text { Per cent of study } \\
\text { population }\end{array}$ \\
\hline Paranoid & 23 & 64 \\
Schizoid & 18 & 50 \\
Dissocial & 19 & 53 \\
Emotionally & 18 & 50 \\
$\quad$ unstable & & \\
Histrionic & 12 & 33 \\
Anankastic & 8 & 22 \\
Avoidant & 18 & 50 \\
Dependent & 10 & 28 \\
\hline
\end{tabular}

of an association with a known aetiological factor. Severe psychosocial adversity in childhood (especially physical and sexual abuse) is an acknowledged risk factor for antisocial, borderline and histrionic personality disorder in people without learning disability. These diagnoses frequently co-occur and, following their study of an in-patient psychotherapy service for people referred with clinical diagnoses of predominantly borderline personality disorder, Dolan et al (1995) suggested that 'severity' (referring to breadth of psychopathology) may be more relevant than individual categories. Owing to the high frequency of multiple SAP diagnoses in the study population, this concept of severity was used for further analysis.

When the case notes of the 36 participants were examined, documentation of childhood abuse was found in 11 cases. These represented instances where it was considered that most mental health professionals would regard the events recorded as unequivocally abusive. For example, one participant had been sexually abused repeatedly by his father and members of the extended family. The perpetrators subsequently were prosecuted. Another had been taken into care as a consequence of physical abuse and evidence of gross physical and emotional neglect.

Odds ratios of prior exposure to early trauma were calculated against two possible adult outcomes: receiving a clinician diagnosis of personality disorder ('antisocial', 'emotionally unstable' or 'histrionic') and a SAP-derived diagnosis of 'severe personality disorder'. For the latter, an arbitrary cut-off was chosen for severity: participants with five or more personality disorder diagnoses were rated as having 'severe personality disorder'. This threshold was decided upon prior to data analysis and 14 participants were rated as having 'severe personality disorder' $(38 \%$ of the sample). The results are shown in Table 2.

\section{Overlap of clinician and SAP diagnoses of personality disorder}

Overall, 14 individuals were rated as having severe personality disorder with the SAP. Only five of these had been diagnosed by their psychiatrist as having a personality disorder of any type. In no case where a clinical diagnosis of personality disorder was recorded was more than one category assigned.

Of the other nine individuals, four were diagnosed with a psychotic illness, two with an affective disorder and two with a pervasive developmental disorder (autistic continuum disorder). One of the 'severe' group had received a clinical diagnosis of organic personality disorder. In terms of the validating criterion of early traumatic experience, two of the individuals diagnosed with psychosis, one of those with pervasive developmental disorder, and the person with organic personality disorder had been exposed to abuse when young.

\section{DISCUSSION}

This is a small-scale preliminary study of the prevalence of personality disorder in a learning disabled population with severe behavioural problems whose difficulties were of a nature or degree that could not be met by community-based services. It forms part of a wider body of local work concerning the future development of mental health services, especially against the backdrop of the recently published White Paper dealing with mainstream access to services for people with learning disabilities (Department of Health, 2001).

\section{Prevalence of personality disorder and the utility of the SAP}

The finding that $19 / 36$ participants $(53 \%)$ could be diagnosed with dissocial personality disorder and 18/36 (50\%) with emotionally unstable personality disorder is remarkably in keeping with Ballinger \& Reid's (1987) conclusion that $50 \%$ of their hospital population had the broadly equivalent 'explosive personality disorder' (according to the older ICD-9 categories in use at the time; World Health Organization, 1978). This is a slightly larger group than the $38 \%$ with severe personality disorder because a minority of these individuals did not exceed the severity threshold. From the point of view of methodology, this finding is encouraging. However, it needs to be considered in the context of several issues concerning the diagnosis of personality disorder in this population using the SAP.

There have been only a handful of published systematic studies of personality disorder in learning disability. In the first of these, Ballinger \& Reid (1987) recommended the use of the SAP because it had acceptable interrater reliability in their setting and avoided the problem of subjects

Table 2 Relative risk of exposure to early traumatic experience resulting in a clinical diagnosis of antisocial, emotionally unstable or histrionic personality disorder and a Standardised Assessment of Personality (SAP) rating of 'severe personality disorder'

\begin{tabular}{|c|c|c|c|c|}
\hline & \multicolumn{2}{|c|}{$\begin{array}{c}\text { Clinical diagnosis of personality } \\
\text { disorder }\end{array}$} & \multicolumn{2}{|c|}{$\begin{array}{c}\text { SAP diagnosis of severe personality } \\
\text { disorder }\end{array}$} \\
\hline & Present & Absent & Present & Absent \\
\hline Abuse present & 6 & 5 & 9 & 2 \\
\hline Abuse absent & 5 & 20 & 5 & 20 \\
\hline \multirow[t]{4}{*}{ Odds ratio } & \multicolumn{2}{|c|}{6.3} & \multicolumn{2}{|c|}{13.3} \\
\hline & \multicolumn{2}{|c|}{ Significant $^{\prime}$} & \multicolumn{2}{|c|}{ Significant ${ }^{\prime}$} \\
\hline & \multicolumn{2}{|c|}{$C l=I .3-3 \mid . I$} & \multicolumn{2}{|c|}{$\mathrm{Cl}=2.2-80.5$} \\
\hline & \multicolumn{2}{|c|}{$P=0.039$} & \multicolumn{2}{|c|}{$P=0.005$} \\
\hline
\end{tabular}

I. Statistical significance calculated using Fisher's exact test. 
with severe communication impairments having to complete self-report questionnaires or take part in unmodified structured interviews. Subsequent work by these and other investigators have used the same assessment method and the SAP seems to have acquired the status of 'industry standard'. This means that it is reasonably easy to compare the outcomes of different studies but there is the obvious drawback that there have been no serious efforts to compare the SAP's performance in learning disability with schedules taking other perspectives on personality construction or to modify the use of these methods for this group.

Finally, it may be that the SAP itself deserves some modification to preserve its construct validity. For example, it was hard for informants to make confident inferences about complex cognitive constructs such as 'continual feelings of emptiness' (item 2 in the criteria for borderline personality disorder) in people who may not have the ability to formulate and communicate such an idea for themselves. Interpreting the threshold for crossing from personality 'accentuation' to 'disorder' also can be problematic: some informants found it understandably difficult to separate the occupational or social impairments secondary to learning disability from those related to personality dysfunction.

\section{Diagnosis of personality disorder and experience of childhood abuse}

Despite acceptance of the SAP as an important diagnostic instrument in learning disability research, there have been no studies of its validity (and, by extension, the validity of the diagnosis of personality disorder itself) in this group. With this in mind, the study took the opportunity to look at the possible relevance of exposure to psychological trauma to the development of personality disorder in adults with learning disability.

Psychosocial adversity, especially exposure to abuse, in early life is an important risk factor for severe personality disorder in adult life in the general population (Herman et al, 1989). Similarly adversity is common in people with learning disabilities but there have been few attempts systematically to relate experiences of abuse to challenging behaviour, and none to formal psychiatric diagnoses. The calculated odds ratios suggest that, whether diagnosed by clinician or research questionnaire, some presentations of personality disorder in people with mild to moderate learning disabilities share the same risk of prior exposure to early psychosocial adversity.

The magnitude of the risk is uncertain, with the small sample size leading to large confidence intervals. However, the association certainly seems more robust for severe personality disorder diagnosed by the SAP than it does for the clinical diagnosis of personality disorder. Unfortunately, the overlap of large confidence intervals prevents any definitive conclusion. The possibility of a trend in odds ratio of 13.3 v. 6.3 may give a tentative nod to Dolan et al's (1995) suggestion that breadth of psychopathology is a meaningful idea. At any rate, it does not undermine our strategy of recording multiple diagnoses and stratifying the sample into 'severe' and 'mild/moderate'.

It must be emphasised also that the definition of early trauma was not more closely defined at this stage. It was felt to be premature to narrow abuse down to specific categories, in view of the study's preliminary nature and its 'hypothesisgenerating' intention. It relied on the principal investigator's subjective judgement that some clear-cut event that had at least prompted action by social services had occurred. However, this judgement was made blind to the SAP diagnosis. There are important ethical constraints on pursuing abuse histories for research purposes in learning disability and it is hoped that these results will make it justifiable to pursue this line more systematically in our group in the future. An initial step would be to map 'developmental trajectories' to adult disorder using qualitative methods.

\section{Research versus clinical diagnosis}

The discrepancy between clinical and research diagnoses is interesting. One might conclude that it represents a case of misdiagnosis by clinicians, especially if the result diagnosis looks more 'valid' in terms of exposure to a particular causative factor. However, other standards of validity exist that are, arguably, more relevant to clinical practice, especially response to treatment and prognosis. Also, it may be that clinicians have deeper reservations about diagnosing personality disorder. For example, some have doubts that it is appropriate to make the diagnosis at all in those with severe and profound learning disability
(Gostason, 1985), although others have discussed cases of narcissistic personality disorder in people with IQs of less than 20 (Goldberg et al, 1995). Furthermore, there may be concern, made explicit by one psychiatrist whose patients were surveyed in this study, that diagnosing personality disorder implied 'untreatability' and its connotations were prejudicial in identifying community placements for hospital residents.

\section{Implications of validity studies in learning disability}

Although the association observed here requires confirmation through more rigorous investigation, its demonstration in terms of linkage to a specific diagnosis has important implications. People with learning disabilities are generally excluded from mainstream mental health research. Although this may be partly owing to concerns about capacity to consent, there remains an assumption that behavioural and emotional problems are either organically mediated or environmentally contingent. This is reflected in approaches to challenging behaviour emphasising the 'symptomatic' use of medication, behavioural intervention or both (Emerson, 1995). Recognition that many examples of challenging behaviour are expressions of psychiatric conditions such as psychosis or affective disorder has given a rationale for the use of psychotropic drugs but, importantly, has permitted people with learning disabilities to have access to 'mainstream' treatments. This is a core principle underlying the practice of specialist learning disability psychiatry.

However, it is only recently that access to psychotherapy, especially for traumatised individuals, has become an issue (Hollins \& Sinason, 2000). Psychotherapy is of particular relevance to personality disorder, in various forms, because it constitutes the only intervention with any degree of evidence-based efficacy (e.g. Menzies et al, 1993). This does not mean to say that psychotherapy is appropriate for all adults with learning disabilities who have diagnosable personality disorder, but then neither is it appropriate for all those cases where learning disability is not involved. It does mean that psychotherapies for some adults with lifelong histories of severely challenging behaviour should be a priority for health service research with a view to future service development. 
Demonstrating the validity of personality disorder may serve a wider purpose. Personality disorder research and clinical practice has come to focus particularly on psychotherapy and related themes. Biological, behavioural and, to some extent, social models have received less attention. In a recent review of neuropsychiatric perspectives on personality disorder, Fogel \& Ratey (1995) point out that of all psychiatric diagnoses, disorders of personality are most likely to be seen as 'functional'. Akiskal (1981) has suggested that many cases of severe personality disorder can be seen as presentations of organically mediated affective disorders, but 'misdiagnosis' has denied access to effective pharmacotherapy (or even any service at all). More recently, Linehan $e t$ al (1993) have shown that behavioural principles can underpin effective therapy for women with borderline personality disorder. Paris (1996) offers an important theory of personality disorder in biosocial terms, showing how symptoms can be seen to serve a function in terms of the interaction between the individual and his or her social environment.

Many specialist clinicians in the field of learning disabilities will point out that they have been successfully applying these ideas for some time but have done so under the guise of 'challenging behaviour' rather than personality disorder. It is notable therefore that some important concepts more usually associated with learning disability research have been used to investigate aspects of severe personality disorder. An example is Blair et al's proposal (1996) that aspects of antisocial personality disorder reflect a failure in the ability to develop 'theory of mind', an aspect of cognitive psychology that has played a major part in understanding autism. It may turn out to be the case that general psychiatry can learn from the problems faced by adults with learning disabilities, but one needs to know where to look.

\section{ACKNOWLEDGEMENTS}

We thank Anna Cawdery for her vital support in the preparation of this study and Sheryl Kopel for statistical advice.

\section{REFERENCES}

Akiskal, H. S. (198I) Sub-affective disorders, dysthymic, cyclothymic and bipolar II disorders in the borderline realm. Psychiatric Clinics of North America, 4 $25-46$.

\section{CLINICAL IMPLICATIONS}

- There is preliminary evidence that personality disorder in adults with learning disability is a valid entity, in terms of shared aetiology with the disorder in the general psychiatric population.

- This observation supports arguments for the development of evidence-based treatment services, especially psychotherapy, for adults with learning disability and personality disorder.

- There may be an important 'learning disability perspective' on personality disorder in general, but this is hidden beneath the label of 'challenging behaviour'.

\section{LIMITATIONS}

This is a small-scale study that is unable to clarify the magnitude of the observed association with abuse.

It is restricted to in-patient units and requires replication in community settings.

Other important aspects of validity, particularly outcome and response to intervention, were not examined and require urgent investigation.

ANDREW FLYNN, MRCPsych, HELEN MATTHEWS, MRCPsych, SHEILA HOLLINS, FRCPsych, Department of Psychiatry of Disability, St George's Hospital Medical School, London

Correspondence: Andrew Flynn, Mental Health of Learning Disabilities, Oxleas NHS Trust, c/o 183 Lodge Hill, Goldie Leigh, Abbey Wood, London SE2 OAY, UK

(First received 10 July 200I, final revision 14 January 2002, accepted 17 January 2002)

Ballinger, B. R. \& Reid, A. H. (1987) A standardised assessment of personality disorder in mental handicap. British Journal of Psychiatry, 150, 108-109.

Blair, J., Sellars, C., Strickland, I., et al (1996) Theory of mind in the psychopath. Journal of Forensic Psychiatry, 7, $15-25$.

Department of Health (200I) Valuing People: A New Strategy for the 21st Century. London: HMSO

Dolan, B., Evans, C. \& Norton, K. (1995) Multiple

Axis-Il diagnoses of personality disorder. British journal of Psychiatry, 166, 107-112.

Emerson, E. (1995) Challenging Behaviour: Analysis and Intervention in People with Learning Difficulties. Cambridge: Cambridge University Press.

Fogel, B. S. \& Ratey, J. J. (1995) A neuropsychiatric approach to personality disorder. In Neuropsychiatry of Personality Disorder (ed. J. J. Ratey), pp. I-16. Cambridge, MA: Blackwell Science.

Goldberg, B., Gitta, M. Z. \& Puddephat, A. (1995) Personality and trait disturbances in an adult mental retardation population: significance for psychiatric management. Journal of Intellectual Disability Research, 39. 284-294.

Gostason, R. (1985) Psychiatric illness among the mentally retarded. Acta Psychiatrica Scandinavica, $7 \mathbf{I}$ (suppl. 318), 3-117.

Herman, J., Perry, C. \& Kolk, B. (1989) Childhood trauma in borderline personality disorder. American Journal of Psychiatry, 146, 490-495.
Hollins, S. \& Sinason, V. (2000) Psychotherapy, learning disabilities and trauma: new perspectives. British Journal of Psychiatry, 176, 32-36.

Khan, A., Cowan, C. \& Roy, A. (1997) Personality disorders in people with personality disorders: a community study. Journal of Intellectual Disability Research, 4I, 324-330.

Linehan, M., Heard, H. \& Armstrong, H. (1993) Naturalistic follow-up of a behavioural treatment for chronically suicidal borderline patients. Archives of General Psychiatry, 50, 97I-974.

Menzies, D., Dolan, B. M. \& Norton, K. (1993) Are short term savings worth long term costs? Funding treatment for personality disorders. Psychiatric Bulletin, I7, 517-519.

Paris, J. (1996) Social Factors in the Personality Disorders: A Biopsychosocial Approach to Aetiology and Treatment. Cambridge: Cambridge University Press.

Pilgrim, J. A., Mellers, J.D., Boothby, H. A., et al (1990) Inter-rater and temporal reliability of the Standardised Assessment of Personality and the influence of informant characteristics. Psychological Medicine, 23, 779-786.

World Health Organization (1978) Ninth Revision of the International Classification of Diseases and Related Health Problems (ICD-9). Geneva: WHO.

- (1992) Tenth Revision of the International Classification of Diseases and Related Health Problems (ICD-10). Geneva: WHO. 